\title{
In vitro Biosynthesis of Human Chorionic Follicle Stimulating Hormone
}

\author{
TAKESHI MARUO \\ Department of Obstetrics and Gynecology, \\ Kobe University School of Medicine, Kobe 650
}

\section{Synopsis}

In order to explore the possibility that human chorionic FSH (hCFSH) may be synthesized in vitro by the placenta and secreted into the culture media, chorionic tissue of the first trimester was cultivated in the radioactive medium prepared by adding ${ }^{3} \mathrm{H}$-proline and/or ${ }^{14} \mathrm{C}$-glutamic acid. Purification of biosynthesized hCFSH from the media was carried out by a combination of Sephadex G-100 gel filtration, DEAE-cellulose chromatography and polyacrylamide disc-gel electrophoresis.

Utilizing radioimmunoassay for hCFSH, incorporation of labeled amino acid into biosynthesized hCFSH was examined. ${ }^{3} \mathrm{H}$-proline alone could not be incorporated easily into the biosynthesized hCFSH molecule but was predominantly incorporated into the biosynthesized hCG molecule. In a double labeling experiment with ${ }^{3} \mathrm{H}$-proline and ${ }^{14} \mathrm{C}$-glutamic acid, however, it was certified that hCFSH labeled with both ${ }^{3} \mathrm{H}$-proline and ${ }^{14} \mathrm{C}$-glutamic acid could be synthesized in vitro. Since hCFSH contains less proline and more glutamic acid than hCG, there may be a mutual relationship between the rate of incorporation of labeled amino acid into the hormones and the amino acid composition of the hormones.

In addition, on DEAE-cellulose chromatography immunoreactive hCFSH eluted at a lower conductivity than native hCFSH and its elution profile was consistent, while immunoreactive hCG eluted at a higher conductivity than native hCG and its elution profile demonstrated a considerable variation. These results suggested that hCFSH synthesized in vitro might be stable, whereas hCG synthesized in vitro might exhibit a certain polymorphism due to charge heterogeneity.

It has already been clarified and reported that human chorionic gonadotropin (hCG) with LH-like activity and human chorionic FSH (hCFSH) with FSH-like activity could be isolated separately from the chorionic tissue (Tojo, 1968 ; Ashitaka, 1970; Tojo et al., 1973). Protein hormone synthesis by placenta is not well understood yet although there have been several reports available on the biosynthesis of hCG (Benagiano et al., 1972 ; Patrito et al., 1973 ; Tojo et al., 1974; Maruo et al., 1974) and on human placental lactogen (Friesen et al., 1968). In the pre-

Received for publication November 4, 1975. liminary reports, the author et al. demonstrated that ${ }^{3} \mathrm{H}$-labeled hCG was biosynthesized in vitro in a culture system of chorionic tissue using ${ }^{3} \mathrm{H}$-proline as a radioactive precursor, and it had some different conformational changes in comparison with native hCG. Simultaneously we found that the concentrated medium in a culture system of chorionic tissue showed an immunological cross-reactivity with anti-hCFSH serum. These findings prompted us to study the incorporation in vitro of labeled amino acid into hCFSH.

In the work reported here we tried to achieve radioimmunoassay of $\mathrm{hCFSH}$, and 
then the biosynthesis of hCFSH in placenta was followed by using this radioimmunoassay system.

\section{Materials and Methods}

The purified hCFSH $(2,200 \mathrm{IU} / \mathrm{mg}$ equivalent, by an ovarian weight method) was prepared from lyophilized powders of trophoblastic tissue of normal pregnancy in the first trimester by the methods reported previously (Ashitaka, 1970).

Antiserum to hCFSH was obtained by repeated injections of this hormone into guinea pigs in a manner similar to that described by Wide and Gemzell (1969). Antibodies to nonspecific components were removed by repeated absorptions with lyophilized normal male serum protein and purified hCG. This serum developed only one precipitation line on agar gel diffusion.

Iodination of hCFSH was carried out by the enzymatic iodination method described in detail elsewhere (Ashitaka \& Koide, 1974). The iodinated hCFSH was separated from unreacted $\mathrm{Na}^{125} \mathrm{I}$ by gel filtration on Bio-Gel P-60 column $(1 \times 24 \mathrm{~cm})$ which had been presaturated with albumin. The average specific radioactivity of ${ }^{125 I-h C F S H}$ was $65-$ $70 \mu \mathrm{Ci} / \mu \mathrm{g}$. In order to examine whether any denaturation took place in the process of the radioiodination or not, the iodinated hormone was subjected to polyacrylamide disc-gel electrophoresis. The distribution of ${ }^{125}$ I-radioactivity in the disc-gel was examined in comparison with the elution pattern of the purified hCFSH. The radioactivity was measured in a Shimazu automatic gamma counter, set to count ${ }^{125} \mathrm{I}$.

\section{Radioimmunoassay:}

The radioimmunoassay was performed by the double-antibody technique (Tojo et al., 1969). Fig. 1 shows an outline of the assay procedures. AntihCFSH serum was used in a final concentration of $1: 12,000$ and the bound percentage of labeled hormone to the antiserum was $15-20$ at this concentration. Repeated studies confirmed that these conditions were found to give equally good results. The second antibody (anti-guinea pig $\gamma$-globulin of rabbit) was supplied by Dr. U. Koga of Shimizu Pharmaceutical Laboratory, Shizuoka, Japan.

Chorionic tissue culture:

Human chorionic tissue was obtained at the time of therapeutic abortion by curettage in the first trimester of pregnancy. Organ culture of chorionic tissue was carried out for 5 days in a manner similar to that described by the author et al. (1974). $50 \mathrm{mg}$ of wet weight of minced placental fragments was placed on filter paper $(25 \mathrm{~mm}$ in diameter $)$ within a glass Petri dish ( $70 \mathrm{~mm}$ in diameter). $15 \mathrm{ml}$ of culture medium per one dish was then added.
Incubations were performed at $37^{\circ} \mathrm{C}$ under a $95 \%$ $\mathrm{O}_{2}-5 \% \mathrm{CO}_{2}$ atmosphere. The culture medium used was prepared by adding $1 \mu \mathrm{Ci}$ of ${ }^{3} \mathrm{H}$-proline (519 $\mathrm{mCi} / \mathrm{mmol}$, Amerscham) and /or $1 \mu \mathrm{Ci}$ of ${ }^{14} \mathrm{C}$-glutamic acid $(280 \mathrm{mCi} / \mathrm{mmol}$, New England Nuclear) per $\mathrm{m} l$ of a synthetic medium " 199 " containing penicillin $(100 \mathrm{IU} / \mathrm{ml})$ and streptomycin $(100 \mu \mathrm{g} / \mathrm{ml})$.

Extraction and purification of biosynthesized hCFSH:

The method of extraction and purification of hCFSH and hCG from the culture medium, previously described (Maruo et al., 1974), is essentially as follows: at the end of culture pooled medium for a total of $500 \mathrm{mg}$ of chorionic tissue was concentrated into about $5 \mathrm{ml}$ in a rotatory vacuum evaporator and was then filtered through a Sephadex G-100 column $(2 \times 90 \mathrm{~cm})$, equilibrated and eluted at $4^{\circ} \mathrm{C}$ with $0.005 \mathrm{M}$ Tris- $\mathrm{HCl}$ buffer, $\mathrm{pH}$ 8.6. Fractions of $4.8 \mathrm{ml}$ were collected. After the gel filtration the radioactive protein fraction was chromatographed on DEAE-cellulose column $(2 \times 15 \mathrm{~cm})$, equilibrated in the same condition, and was eluted by a linear gradient elution at $0.4 \mathrm{M}$ Tris- $\mathrm{HCl}$ buffer, $\mathrm{pH}$ 8.6. The elution of proteins was checked by ultraviolet absorption at $280 \mathrm{~m} \mu$ with a Shimazu QV-50 spectrophotometer. Each fraction was measured for ${ }^{3} \mathrm{H}$ - and ${ }^{14} \mathrm{C}$-radioactivity in a Packard Tri-Carb liquid scintillation spectrophotometer and was assayed in radioimmunoassays for $\mathrm{hCFSH}$ and $\mathrm{hCG}$.

Fractions on DEAE-cellulose chromatography which had hCG or hCFSH activity were respectively submitted to polyacrylamide disc-gel electrophoresis by the technique of Orstein (1964) and Davis (1964). The columns were sliced transversely into segments of a thickness of $2.4 \mathrm{~mm}$ as previously reported (Maruo et al., 1974). Each segment was measured

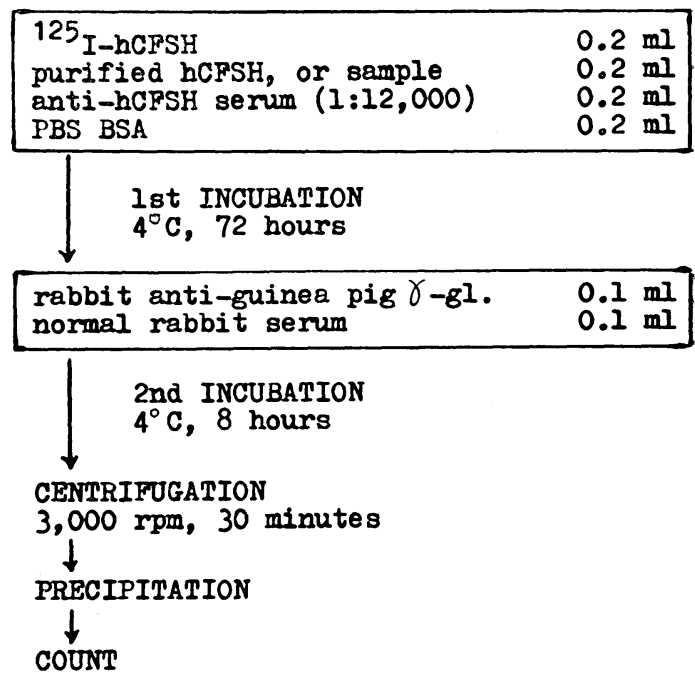

Fig. 1. Procedure of radioimmunoassay for hCFSH. 
for ${ }^{3} \mathrm{H}$ - and ${ }^{14} \mathrm{C}$-radioactivity and also for $\mathrm{hCG}$ and hCFSH immunoreactivity.

\section{Results}

\section{Radioiodination :}

When hCFSH iodinated by the enzymatic method was subjected to polyacrylamide disc-gel electrophoresis, ${ }^{125}$ I-radioactivity showed a single peak at segment No. 15 of the disc corresponding to the migration area of native hCFSH (Fig. 2). From this result it was determined that the iodinated hormone was not denatured on the electrophoretic mobility through the radioiodination procedures.

\section{Radioimmunoassay:}

Fig. 3 shows a standard curve for hCFSHradioimmunoassay system. The amount of labeled hCFSH bound to the tubes in the presence of various amounts of unlabeled hCFSH is expressed as a percentage of the radioactivity bound to the control tubes (no unlabeled hCFSH). The sensitivity of the

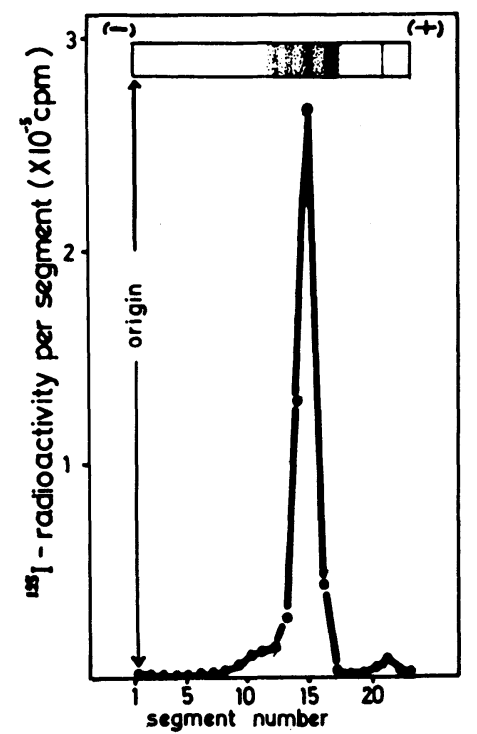

Fig. 2. Pattern in $7 \%$ polyacrylamide disc-gel electrophoresis $(\mathrm{pH} 8.8,0.05 \mathrm{M}$ sodium glycinate buffer) of ${ }^{125} \mathrm{I}-\mathrm{hCFSH}$. CPM vs. transverse gel slices. assay was between 0.01 and $0.02 \mu \mathrm{g} \mathrm{hCFSH/}$ $\mathrm{m} l$. Unlabeled hCG produced no inhibition at the concentration up to $50 \mu \mathrm{g}$ $\mathrm{hCG} / \mathrm{m} l$, but a slight inhibition was observed when the effects of higher concentrations were studied (Fig. 3).

Extraction and purification of the biosynthesized hCFSH:

After chorionic tissues were cultured for 5 days in the radioactive medium which was prepared by adding $1 \mu \mathrm{Ci}$ of ${ }^{3} \mathrm{H}$-proline per $\mathrm{m} l$, a pool of the culture media was applied to the column of Sephadex G-100. The elution profile of the culture media after gel filtration is illustrated in Fig. 4. Maximum hCFSH immunoreactivity was found in fraction No. 14, whereas maximum hCG immunoreactivity was found in fraction No. 13. These protein fractions were closely coincided with the distribution of ${ }^{3} \mathrm{H}$-radioactivity.

The radioactive protein fractions (tubes 10-18) on Sephadex G-100 were further chromatographed on DEAE-cellulose column. Immunoreactivity of hCFSH showed a peak at a conductivity of $5.8 \mathrm{mmhos}$, whereas that of hCG at a conductivity of $4.7 \mathrm{mmhos}$. Simultaneously it was observed that there was a mutual relationship between hCG immunoreactivity and ${ }^{3} \mathrm{H}$-radioactivity. The elution peak of the adsorbed protein on

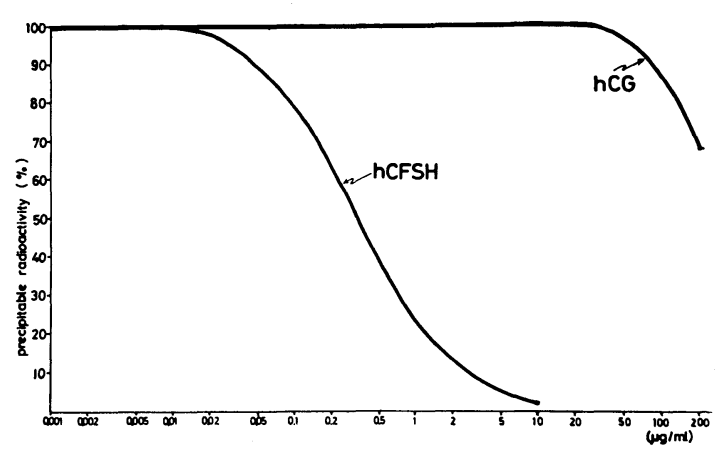

Fig. 3. Standard curve for hCFSH in radioimmunoassay. Unlabeled hCG produced no inhibition at the concentration up to $50 \mu \mathrm{g} / \mathrm{ml}$. 


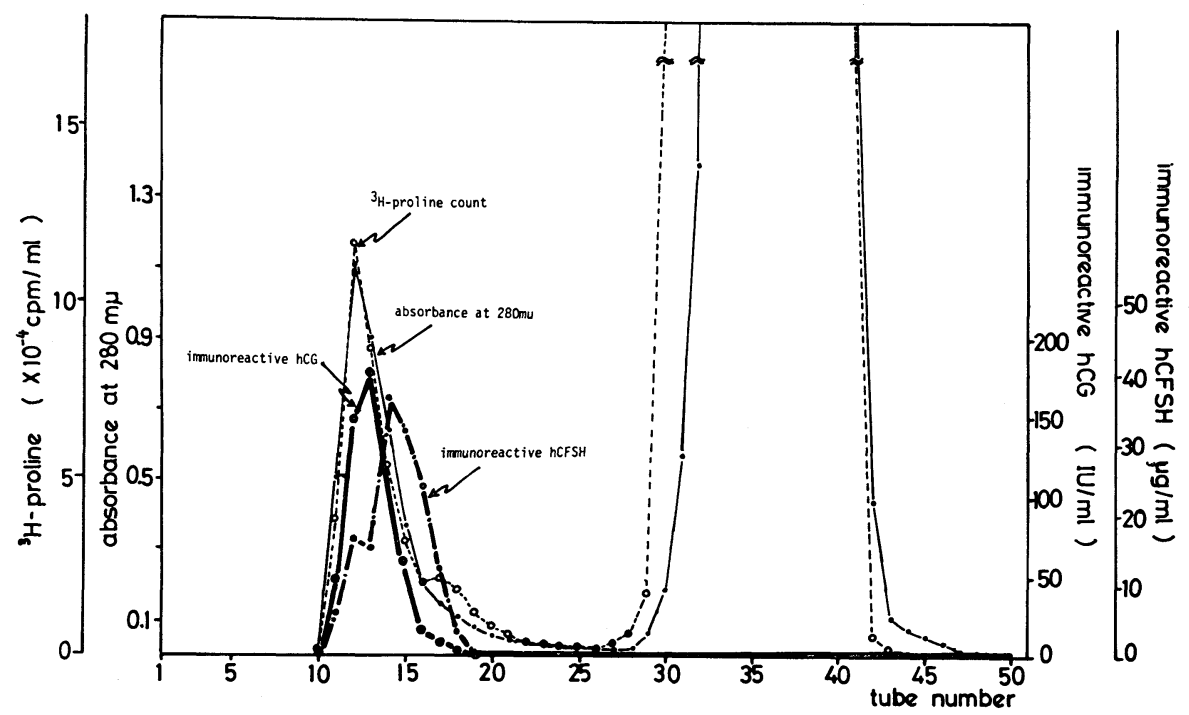

Fig. 4. Gel filtration of the culture medium on Sephadex G-100 (single labeling experiment with ${ }^{3} \mathrm{H}$-proline). Immunoreactive hCFSH peak was apparent in tube $\# 14$, whereas immunoreactive hCG peak was found in tube $\$ 13$. The distribution of ${ }^{3} \mathrm{H}$-radioactivity coincided closely with that of hCG immunoreactivity.

DEAE-cellulose was apparent at a similar conductivity to the area where hCFSH immunoreactivity showed a peak (Fig. 5).

The peak of hCG immunoreactivity on DEAE-cellulose chromatography (conductivity 4.5-4.7 mmhos) and of hCFSH immunoreactivity (conductivity 5.8-6.0 mmhos) were respectively submitted to polyacrylamide disc-gel electrophoresis. As shown in Fig. 6, ${ }^{3} \mathrm{H}$-radioactivity was observed slightly at segment No. 13-15 where the peak of hCFSH immunoreactivity appeared, although ${ }^{3} \mathrm{H}-$ radioactivity and hCG immunoreactivity showed a coincidental sharp peak at segment No. 6-7 on the disc. From these results it was assumed that ${ }^{3} \mathrm{H}$-proline added to the culture medium was little utilized as a precursor of biosynthesized hCFSH.

Fig. 5. Chromatography of the radioactive protein on DEAE-cellulose. The adsorbed protein was eluted by linear gradient elution method. The abscissa indicates conductivity. Immunoreactivity of hCFSH showed a peak at a conductivity of $5.8 \mathrm{mmhos}$, whereas hCG immunoreactivity showed a peak at a conductivity of $4.7 \mathrm{mmhos}$ with a coincidental distribution of ${ }^{3} \mathrm{H}$-radioactivity.

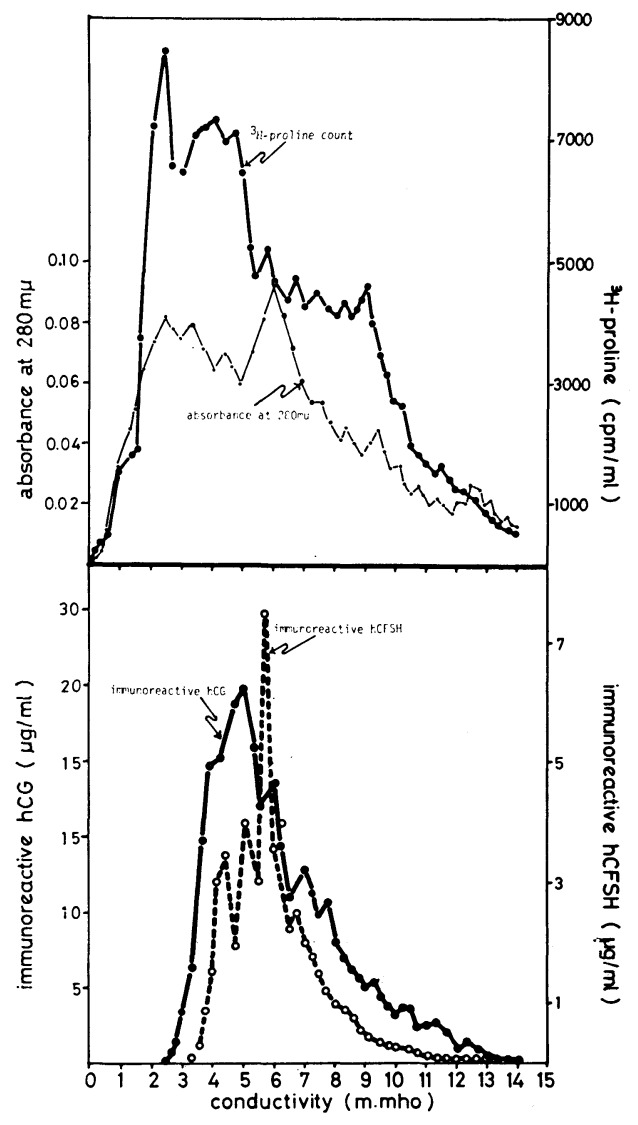



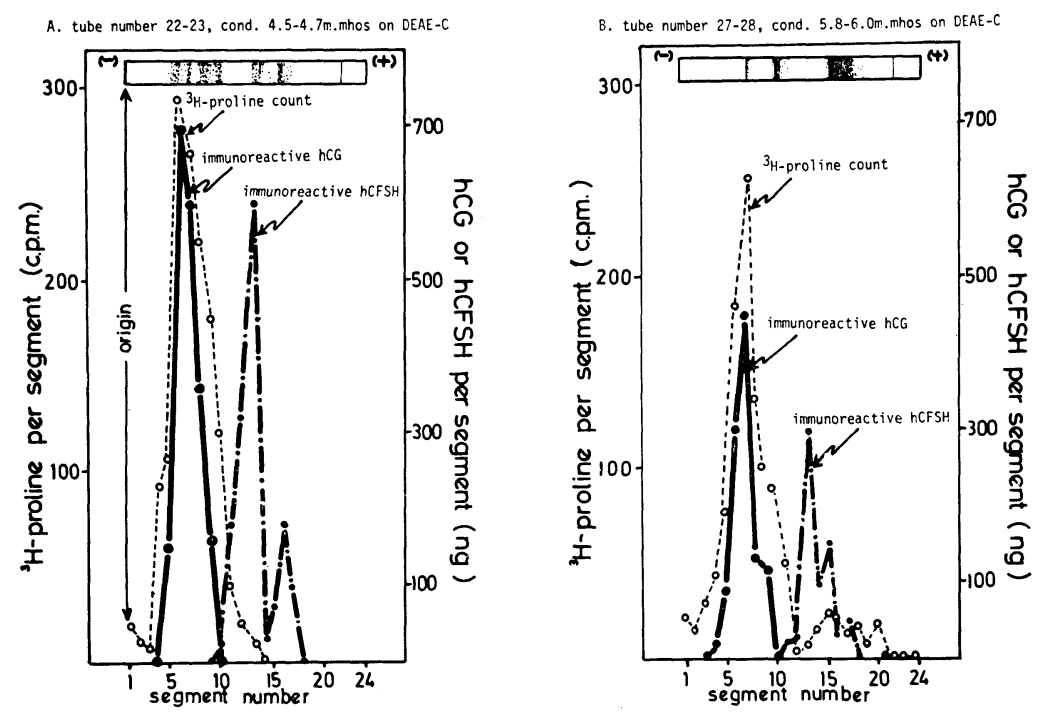

Fig. 6. Distribution of ${ }^{3} \mathrm{H}$-radioactivity and of immunoreactivity of hCG and hCFSH after polyacrylamide disc-gel electrophoresis of protein fractions on DEAE-cellulose. ${ }^{3} \mathrm{H}$-radioactivity was slightly recognized at segment \#13-15 where hCFSH immunoreactivity was admitted. Immunoreactivity of hCG showed a sharp peak coincidental with ${ }^{3} \mathrm{H}$-radioactivity at segment $\# 6-7$.

In the next study, in order to confirm the biosynthesis in vitro of labeled hCFSH, ${ }^{14} \mathrm{C}$-glutamic acid was used as a marker of biosynthesized hCFSH because of the high concentration of glutamic acid in the hCFSH molecule (Ashitaka, 1970). Chorionic tissues were cultured for 5 days in the radioactive medium which was prepared by adding $1 \mu \mathrm{Ci}$ of ${ }^{3} \mathrm{H}$-proline and of ${ }^{14} \mathrm{C}$-glutamic acid per $\mathrm{m} l$. When a pool of the cultured media was filtered through the Sephadex G-100 column, hCG and hCFSH immunoreactivity showed peaks in fraction No. 14 and 15 respectively with coincidental peaks of ${ }^{3} \mathrm{H}$ - and ${ }^{14} \mathrm{C}$-radioactivity whose elution patterns were similar (Fig. 7).

The radioactive protein fractions (tubes 10-19) on Sephadex G-100 were admitted to DEAE-cellulose chromatography. As illustrated in Fig. 8, hCFSH immunoreactivity was found at a conductivity of 3.5-7.0 mmhos, whereas hCG immunoreactivity was found at a conductivity of 8.0-14.0 mmhos. ${ }^{3} \mathrm{H}$ - and ${ }^{14} \mathrm{C}$-radioactivity respectively showed

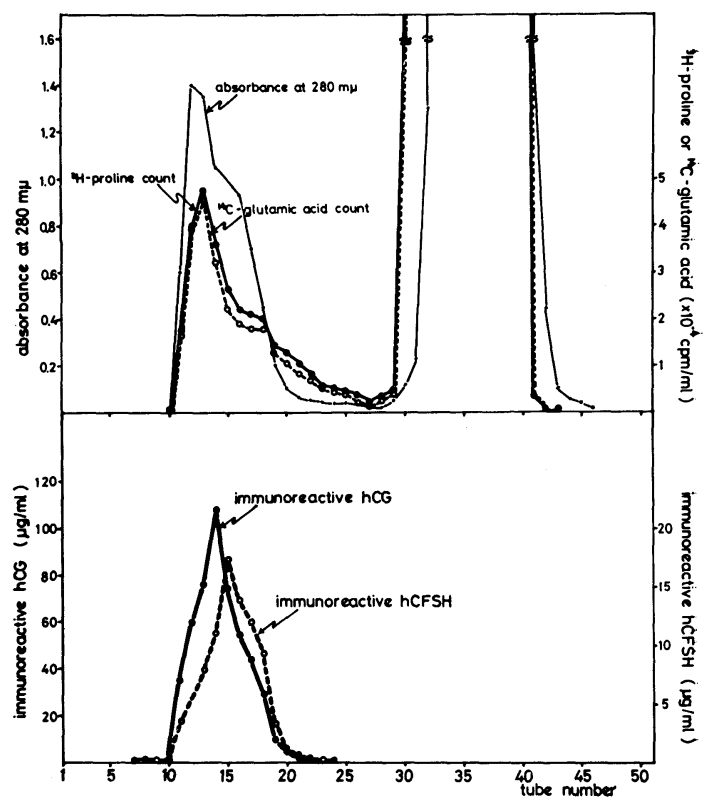

Fig. 7. Gel filtration of the culture medium on Sephadex G-100 (double labeling experiment with ${ }^{3} \mathrm{H}$-proline and ${ }^{14} \mathrm{C}$-glutamic acid). Immunoreactive hCFSH peak was found in tube $\sharp 15$, whereas immunoreactive hCG peak appeared in tube $\$ 14$. The distributions of both ${ }^{3} \mathrm{H}$ - and ${ }^{14} \mathrm{C}$-radioactivity were similar. 
similar distributions which mostly corresponded to that of hCG immunoreactivity. The elution pattern of immunoreactive hCFSH was similar to that of adsorbed protein checked by ultraviolet absorption at $280 \mathrm{~m} \mu$.

Immunoreactive hCFSH fraction (conductivity 3.5-6.5 mmhos) and hCG fraction (conductivity 8.5-10.5 mmhos) on DEAEcellulose were respectively submitted to polyacrylamide disc-gel electrophoresis. In the disc of the former fraction hCFSH immunoreactivity showed a peak at segment No. 12 with a coincidental peak of ${ }^{3} \mathrm{H}$ - and ${ }^{14} \mathrm{C}$ radioactivity, whereas hCG immunoreactivity was found only slightly at segment No. 7 (Fig. 9). On the other hand, in the disc

Fig. 8. Chromatography of the radioactive protein on DEAE-cellulose. The adsorbed protein was eluted by linear gradient elution method. The abscissa indicates conductivity. Immunoreactivity of hCFSH was found at a conductivity of 3.5-7.0 mmhos and showed a peak at a conductivity of $5.6 \mathrm{mmhos}$, whereas hCG immunoreactivity was found at a conductivity of $8.0-14.0 \mathrm{mmhos}$. Both ${ }^{3} \mathrm{H}$ - and ${ }^{14} \mathrm{C}$-radioactivity eluted in a similar pattern mostly corresponded to hCG immunoreactivity.

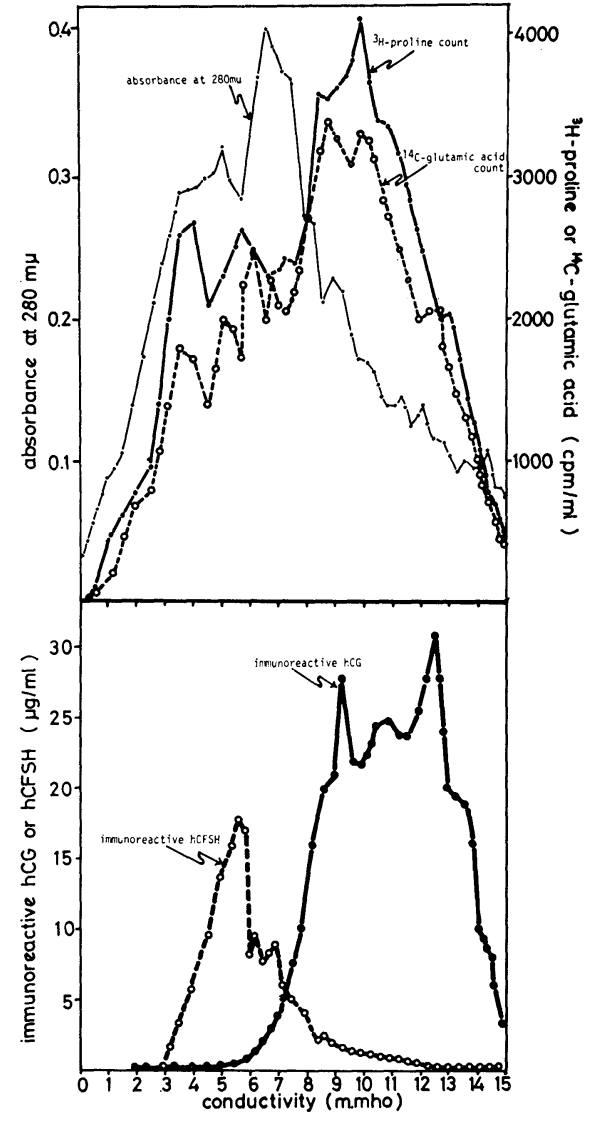

Fig. 9. Distribution of ${ }^{3} \mathrm{H}$ - and ${ }^{14} \mathrm{C}$-radioactivity and of immunoreactivity of hCG and hCFSH after polyacrylamide disc gel electrophoresis of protein fractions on DEAEcellulose. hCFSH immunoreactivity showed a peak at the segment \#12-14 with coincidental peaks of ${ }^{3} \mathrm{H}$ and ${ }^{14} \mathrm{C}$-radioactivity. $\mathrm{hCG}$ immunoreactivity showed a peak at segment $\$ 8$ mostly accompanied with ${ }^{3} \mathrm{H}$-radioactivity.
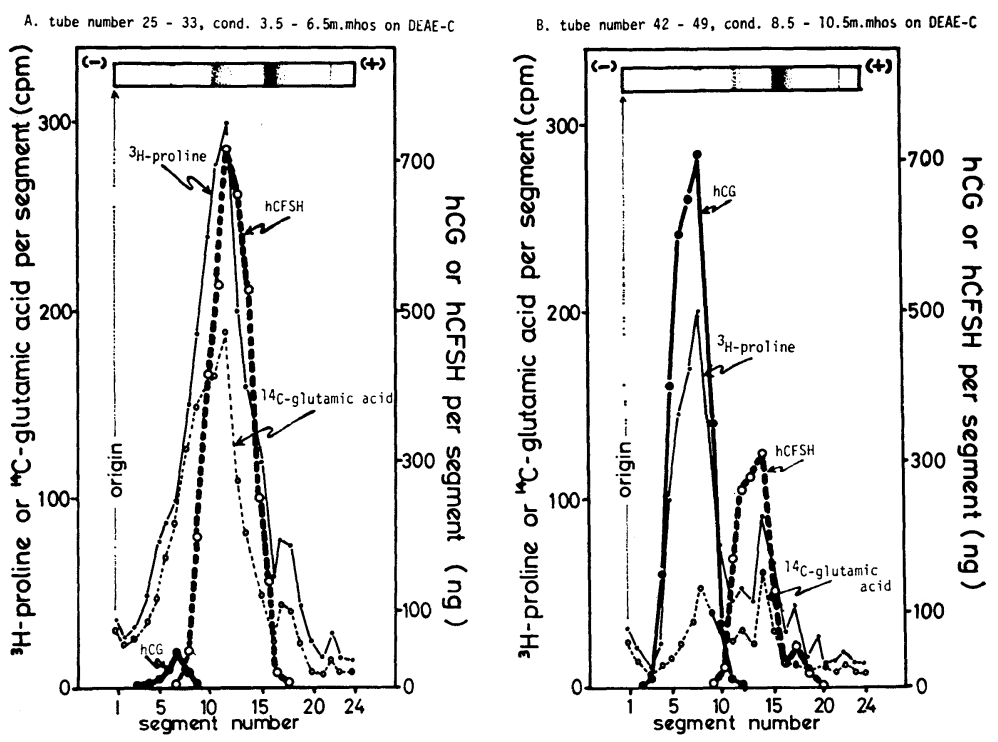
of the latter fraction hCG immunoreactivity and ${ }^{3} \mathrm{H}$-radioactivity showed a coincidental peak at segment No. 8, and simultaneously a small peak of hCFSH immunoreactivity was found at segment No. 14 accompanied by small peaks of ${ }^{3} \mathrm{H}$ - and ${ }^{14} \mathrm{C}$-radioactivity (Fig. 9). These results indicated that hCG labeled with ${ }^{3} \mathrm{H}$-proline was not only biosynthesized but also hCFSH labeled with ${ }^{3} \mathrm{H}$-proline and ${ }^{14} \mathrm{C}$-glutamic acid was biosynthesized in this double labeling experiment.

\section{Discussion}

It was formerly demonstrated that hCG with LH-like activity and hCFSH with FSHlike activity were isolated separately from lyophilized powder of trophoblastic tissue (Ashitaka, 1970; Tojo et al., 1973). Recently the biosynthesis in vitro of hCG from placenta was also confirmed using ${ }^{3} \mathrm{H}$-proline as a radioactive precursor (Maruo et al., 1974). Up to this time, however, there has been no report which detected the presence of radioimmunological FSH activity in the medium of tissue culture of chorionic tissue although some workers have tried to assay chorionic FSH activity by radioimmunoassay for pituitary FSH (Benagiano et al., 1972). The chorionic FSH activity in the cultured medium should be detected by anti-hCFSH serum since hCFSH did not cross-react with anti-FSH serum of pituitary origin in Ouchterlony immunodiffusion (Ashitaka et al., 1970). The radioimmunoassay with anti-hCFSH serum described in the present work provides a sensitive method for the determination of hCFSH. As shown in Results, radioiodination by the enzymatic method did not alter the electrophoretic mobility of hCFSH, and labeled hCFSH appeared to be homogeneous on polyacrylamide disc-gel electrophoresis. The present experiments were performed in order to confirm the biosynthesis in vitro of hCFSH from human placenta by utilizing this radioimmunoassay system.

First, ${ }^{3} \mathrm{H}$-proline was used as a marker of biosynthesized hCFSH. After polyacrylamide disc-gel electrophoresis of the radioactive protein fractions on DEAE-cellulose chromatography, ${ }^{3} \mathrm{H}$-radioactivity was not much observed at the position of the disc where hCFSH immunoreactivity was detectable, but it showed a coincidental sharp peak with hCG immunoreactivity at the migration area of native hCG. This finding suggested that ${ }^{3} \mathrm{H}$-proline alone could not be incorporated easily into the biosynthesized hCFSH molecule, but most of it could be incorporated into the biosynthesized hCG molecule. These results were somewhat to be expected since hCG contains more proline and less acidic amino acid than hCFSH.

From the chemical characteristics of hCFSH which is rich in such acidic amino acid as glutamic acid and aspartic acid, ${ }^{14} \mathrm{C}$-glutamic acid was selected as a marker of biosynthesized hCFSH. After chorionic tissues were cultured for 5 days in the radioactive medium prepared by adding $1 \mu \mathrm{Ci}$ of ${ }^{3} \mathrm{H}$-proline and of ${ }^{14} \mathrm{C}$-glutamic acid per $\mathrm{m} l$, isolation of the biosynthesized hCFSH labeled with ${ }^{14} \mathrm{C}$-glutamic acid from the cultured medium was attempted. On gel filtration and DEAE-cellulose chromatography, ${ }^{3} \mathrm{H}$ - and ${ }^{14} \mathrm{C}$-radioactivity showed similar elution profiles. However, on polyacrylamide disc-gel electrophoresis ${ }^{3} \mathrm{H}$ - and ${ }^{14} \mathrm{C}$-radioactivity showed different distributions in the disc. ${ }^{3} \mathrm{H}$-radioactivity in the disc was not only found at the position of a peak of hCG immunoreactivity but also at the position of a peak of hCFSH immunoreactivity. On the other hand, ${ }^{14} \mathrm{C}$-radioactivity in the disc was mostly detected at the position where hCFSH immunoreactivity appeared. These results suggested that hCG labeled with ${ }^{3} \mathrm{H}$-proline and hCFSH labeled with both ${ }^{3} \mathrm{H}$-proline and ${ }^{14} \mathrm{C}$-glutamic acid were biosynthesized in vitro simultaneously. It was assumed that there might be a mu- 
tual relationship between the rate of incorporation of labeled amino acid into these hormones and the amino acid composition of these hormones.

The fact that hCG and hCFSH labeled with the radioactive precursors are obtained in a culture system of chorionic tissue supports the evidence that placenta is able to synthesize hCG and hCFSH. The author et al. described in their preliminary report (1974) that biosynthesized hCG was more tightly bound to anion exchanger than was native $\mathrm{hCG}$, as also confirmed in the work reported here. However, the situation with regard to biosynthesized hCFSH was different. The biosynthesized hCFSH was found at a conductivity of 3.5-7.0 mmhos, while native hCFSH was eluted at a conductivity of 6.0-8.5 mmhos, suggesting that the biosynthesized hCFSH was more weakly bound to the anion exchanger than was native hCFSH and that it might have different structures or conformational changes from the native hormone as well. These results are consistent with Kohler and his co-workers' observation that a highly purified preparation of urinary hCG and hCG synthesized in vitro by clonal lines of a choriocarcinoma differ in net charge. It is, however, still unknown why such a reverse elution attitude on DEAE-cellulose was caused between hCG and hCFSH biosynthesized in vitro. On the other hand, immunologic hCFSH consistently appeared at the same conductivity of 5.0-6.0 mmhos on DEAE-cellulose chromatography where the elution peak of the adsorbed protein was apparent, although immunologic hCG demonstrated a considerable variation in the elution attitude on DEAE-cellulose as illustrated in Fig. 5 and 8. These findings may suggest that the biosynthesized hCFSH might be more stable than the biosynthesized hCG, which might exhibit a certain polymorphism due to charge heterogeneity observed during DEAE-cellulose chromatography. Graesslin et al. (1973) reported that the charge heterogeneity of purified urinary hCG was probably due to variations in sialic acid content. It is, however, still unknown what accounts for the polymorphism of hCG synthesized in vitro. Further studies will be necessary to elucidate this phenomenon.

\section{Acknowledgements}

At the completion of this paper the author wishes to express his sincere gratitude to Prof. S. Tojo of the Department of Obstetrics and Gynecology, Kobe University School of Medicine for his warm and consistent encouragement throughout this study. The author also sincerely thanks Dr. M. Mochizuki and Dr. Y. Ashitaka of the same department for their kind guidance and helpful suggestions. Finally, the author would like to thank Dr. U. Koga of Shimizu Pharmaceutical Laboratory (Japan) who prepared the anti-hCG and anti-hCFSH serum used in this study.

\section{References}

Ashitaka, Y. (1970). Acta Obst. et Gynec. Jap. 17, 124.

Ashitaka, Y. and S.S. Koide (1974). Fertility and Sterility 25, 177.

Benagiano, G., A. Para, M. Meirinho and M. Ermini (1972). J. Endocr. 55, 387.

Davis, B.J. (1964). Ann. N.Y. Acad. Sci. 121, 404.

Friesen, H.G. (1968). Endocrinology 83, 744.

Graesslin, D., H.C. Weise and W. Braendle (1973). FEBS Lett. 31, 214.

Kohler, P.O., W.E. Bridson, J.M. Hammond, B. Weintraub, M.A. Kirschner and D.H. Van Thiel (1971). Transactions of the Third Karolinska Symposium on Research Methods in Reproductive Endocrinology, Geneva, p. 137.

Maruo, T., Y. Ashitaka, M. Mochizuki and S. Tojo (1974). Endocrinol. Japon. 21, 499.

Orstein, L. (1964). Ann. N.Y. Acad. Sci. 121, 321.

Patrito, L.C., A. Flury, J. Rosato and A. Martin (1973). Hoppe-Seyler's Z. Physiol. Chem. 354, 1129.

Tojo, S. (1968). Human Chorionic Gonadotropin: Report on the Theme Commissioned by Japanese Obst. and Gynec. Soc. (in Japanese with English synopsis).

Tojo, S., M. Mochizuki, K. Tokura and T. Mizusawa (1969). Clin. Endocr. (in Japanese) 17, 523.

Tojo, S., M. Mochizuki and T. Maruo (1974). Gonadotropins and Gonadal Function, Moudgal, N.R. ed. Academic Press, New York, 321. 
Tojo, S., M. Mochizuki and Y. Ashitaka (1973). Amsterdam.

Endocrinology (International Congress Series No. Wide, L. and C.A. Gemzell (1960). Acta Endocr. 273) R.O. Scow ed. p. 605, Excerpta Medica, (Copenhagen) 35, 261. 